\title{
Contribuciones del asesoramiento a la función del docente en la universidad
}

Contributions of the Advice to the Teaching Role in the College

Artículo de investigación | Research paper

Fecha de recepción: 14 de septiembre de 2018

Fecha de aceptación: 10 de junio de 2019

Fecha de disponibilidad en línea: julio de 2020

doi:10.11144/Javeriana.m13.cafd

María José Pérez-Cabrera mariajoseperez@santcugat.cat Universidad de BARCELONA, España

(D) ORCID: https://orcid.org/0000-0001-5265-9833

JuAn Llanes-Ordoñez juanllanes@ub.edu

UniversidAd de BARCELONA, EsPaña

D ORCID: https://orcid.org/0000-0002-0059-9741

Para citar este artículo | To cite this article Pérez-Cabrera, M. J. \& Llanes-Ordoñez, J. (2020). Contribuciones del asesoramiento a la función del docente en la universidad. magis, Revista Internacional de Investigación en Educación, 13, 1-26. doi: 10.11144/Javeriana.m13.cafd 


\title{
Resumen
}

El artículo explora la figura del docente universitario desde un papel próximo al asesoramiento para facilitar la gestión personal de la carrera y la posterior inserción profesional de los estudiantes. La investigación se sitúa dentro de un enfoque cualitativo y comprensivo, con el doble objetivo de identificar necesidades formativas docentes y definir el papel de profesorasesor. La información fue extraída de un grupo de discusión y de una entrevista semiestructurada hecha a docentes, asesores y egresados. El estudio destaca la necesidad de reforzar la relación didáctica y el vínculo del docente con la profesión. También se define el perfil del profesor-asesor universitario.

\section{Palabras clave}

Asesoramiento; docente; orientación profesional; universidad

\begin{abstract}
This article explores the figure of the college teacher from a role next to the advisor. It is intended to make easier the Personal Career Management and the subsequent professional insertion of the students. The research is situated as a qualitative and comprehensive approach with a double aim to identify the formation needs of the teachers and to define the role teacher-advisor. The information has been taken from the discussion group and the semi-structure interview to teachers, advisors and graduates. This study underlines the need to strengthen both the didactic relationship and the link between teachers and their profession. This work also defines the profile for the college teacher-advisor.
\end{abstract}

\section{Keywords}

Counselling; teachers; vocational guidance; universities 
Descripción del artículo | Article description

Artículo de investigación derivado de la simbiosis de dos tesis doctorales realizadas por los firmantes del artículo,

La inserción profesional y gestión de la carrera de titulados

en pedagogía y El asesoramiento de proceso como modelos

de formación inicial del profesorado universitario.

\section{Introducción}

Desde la Declaración de Bolonia, en el año 1999, las universidades europeas han seguido, con ritmos diferentes, un proceso que pretende incrementar la movilidad, la transferencia y el reconocimiento académico (Llanes, Figuera \& Torrado, 2017a), por medio de un enfoque basado en competencias, en el que el estudiantado sea el núcleo articulador de toda acción formativa, de manera que se potencie el desarrollo de su autonomía y su responsabilidad, se mejore su empleabilidad y se facilite la transición y adecuación al mercado de trabajo (Llanes, Figuera \& Torrado, 2015).

En coherencia con este planteamiento, el proyecto del Espacio Europeo de Educación Superior (EEES) asume como objetivos: a) impulsar un cambio de paradigma que facilite el aprendizaje autónomo, b) impulsar la eliminación de barreras a la libre circulación de estudiantes y titulados en Europa, c) hacer de Europa un lugar más atractivo para estudiar y d) incrementar el número de titulados (mejorando los niveles de empleabilidad descritos en la Estrategia Europea 2020) (Comisión Europea, 2010). En este marco, la universidad se redescubre, cimentándose en tres pilares que dan sentido a su misión: a) la investigación: creación conocimiento, b) la docencia: transmisión de conocimiento y c) el desarrollo socioeconómico: fomento de la innovación (Pérez \& Serrano, 2012).

Una de las primeras consecuencias de estos procesos es la redefinición de la universidad a partir de una nueva mirada sobre la profesionalidad. El cambio en la formación de profesionales se ha producido al integrar en esta las competencias específicas del campo profesional y las competencias transversales, permitiendo la adaptación de las personas a nuevos contextos y funciones del mercado laboral. A este proceso de ajuste entre las necesidades del mercado laboral y la formación del colectivo universitario, autores como Cardenal (2006) lo han denominado la legitimación del sistema educativo. Y es en dicha legitimación donde la orientación puede erigirse en una herramienta altamente eficaz (Romero \& Figuera, 2016). 


\section{La orientación académica y profesional del estudiante universitario}

La orientación académica y profesional debe encaminar al alumnado al aprendizaje que desarrollará a lo largo de su vida, la cual, según el Proceso de Bolonia, comprende tres dimensiones clave: personal, académica y profesional. Michavila \& Parejo (2008, p. 85) señalan que "la filosofía que subyace al Proceso de Bolonia otorga al estudiante un papel central y protagonista, además de considerarlo como socio activo y participativo en la reforma universitaria europea ahora en curso", por lo que se remarca la importancia de su implicación y participación en la vida universitaria, más allá de la obtención de un título (Sotomayor-Echenique, Coloma-Tirapegui, Parodi-Sweis, Ibáñez-Orellana, Cavada-Hrepich \& Gysling-Caselli, 2013).

Con relación a la dimensión personal, las cumbres de Bergen (2005), Londres (2007), Nueva Lovaina (2009), Bucarest (2012) y Yeveran (2015)1 insisten en la idea de que la universidad debe asesorar personalmente al estudiantado y explicitan que para ello deberán crearse servicios de apoyo específicos que acompañen a la toma de decisiones y fomenten programas de asesoría para el desarrollo de la carrera.

Como han destacado Piccolo \& Colquitt (2006) y Cordeiro, Taveira, Neves, Silva, Rodrigues \& Costa-Lobo (2017), es fundamental que los estudiantes aprendan a gestionar sus carreras, identificando qué factores serán óptimos para mejorar su propia empleabilidad. La formación universitaria puede preparar a los futuros profesionales en la gestión de sus transiciones de vida y ayudarle al éxito a lo largo de su trayectoria profesional.

\section{La gestión personal de la carrera (GPC)}

En el marco teórico de la orientación profesional, la gestión personal de la carrera (GPC) emerge en la última década como un constructo básico para comprender y ayudar a las personas a transitar y a insertarse en el mercado de trabajo. Teniendo en cuenta contextos laborales inciertos y profesiones en ocasiones poco definidas, poder analizar y comprender cómo se produce este proceso es importante para crear modelos de intervención ajustados a la realidad. El papel de la GPC es esencial en todo el proceso de aprendizaje del estudiante, así como en el desarrollo de las competencias específicas asociadas: hacer partícipe a la persona en la exploración y construcción de su carrera orientada por proyectos de autoría personal

1 Para más información sobre los comunicados del EEES, véase http://www.eees.es/ es/eees-desarrollo-cronologico 
aumentará sus capacidades de aprendizaje y ayudará a desarrollar el capital humano en el mercado, garantizando, al mismo tiempo, el desarrollo económico, social y ambiental de las sociedades.

En la última mitad del siglo XX surgieron modelos para trabajar de manera efectiva la GPC, como el modelo de Greenhaus \& Callanan (1994), quienes consideran la construcción de la carrera profesional a partir de ciertas variables, como la exploración de la persona (autoconocimiento), el establecimiento de objetivos, su puesta en práctica mediante estrategias de intervención y el análisis del proceso seguido (Llanes, Figuera \& Torrado, 2017b); hasta llegar a Lent \& Brown (2013), quienes plantean un modelo que incide en el proceso que la persona sigue desde el inicio hasta la meta de su trayectoria profesional. En este caso, los elementos intervinientes son: la capacidad de planificación, la toma de decisiones, el desarrollo de objetivos, el autoconocimiento, la capacidad de adaptación, las habilidades sociolaborales, la resiliencia, la proactividad, la actitud positiva, la capacidad de resolver problemas, la identidad profesional, la autoeficacia y la motivación (Arnau, 2013; Llanes et al., 2017b).

La GPC plantea el reto de considerar al estudiante como un agente activo, autodeterminado y capaz de autogestionar su progreso profesional, dotando de significado su construcción de la carrera y poniendo en práctica las estrategias necesarias para alcanzar los objetivos propuestos a lo largo de la vida (Llanes et al., 2017b; Pinto, Taveira \& Llanes, 2015).

\section{La figura del profesor-asesor}

Ante la nueva configuración del papel del estudiante universitario, y bajo el modelo promovido por Lent \& Brown (2013), se dirige la mirada hacia las competencias del docente universitario (Badia-Pujol, 2012; Fraser, 2016; Jarauta-Borrasca \& Medina-Moya, 2009), quien, en un papel de acompañamiento denominado profesor-asesor, debe asumir el encargo de orientar al estudiante en su GPC. Pero, concretamente, ¿qué puede aportar esta figura a los retos y necesidades de la universidad de hoy?

Se puede definir al profesor-asesor como aquel que sabe y conoce lo que hace, con las estrategias y los recursos necesarios para acompañar el cambio y la transición en los demás; poseedor de una alta capacidad de interpretación, con base en la cual fundamentar la intervención, y abierto a la renegociación con el otro, en cuanto a los cambios a introducir y los planteamientos de la relación (Nicastro \& Andreozzi, 2006; Pérez, 2016). Precisamente dicha relación es la base de todo proceso de asesoramiento: la figura de este profesional se construye en el "respeto por el otro, el reconocimiento de su experiencia y su trayectoria, la aceptación de sus modos 
de pensar y de sentir, aun cuando no siempre exista coincidencia con ellos" (Nicastro \& Andreozzi, 2006, p. 136). Asumir esta premisa implica respetar y ajustarse a las necesidades de cada estudiante, como el ritmo de trabajo, la dedicación y la implicación (Montero \& Sanz, 2008). El profesor-asesor, por tanto, debe tomar decisiones relativas a qué acciones emprender y mediante qué estrategias llevarlas a cabo, en función de las inquietudes de sus estudiantes y de la etapa de desarrollo en las que se encuentran (EstévezNenninger, Valdés-Cuervo, Arreola-Olivarría \& Zavala-Escalante, 2014; Hennissen, Crasborn, Brouwer, Korthagen \& Bergen, 2011).

La literatura muestra una amplia e interesante caracterización de la compleja figura del profesor-asesor (Hall, Draper, Smith \& Bullough, 2008; Hennissen et al., 2011; Nicastro \& Andreozzi, 2006; Sánchez-Tarazaga, 2016), quien debe tener confianza en las posibilidades de desarrollo de las personas a las que asesora, mostrar determinadas cualidades personales (como empatía, cordialidad, accesibilidad, flexibilidad y sinceridad), saber comunicar, proporcionar un rapport de calidad y desarrollar la habilidad de la escucha como elemento imprescindible para llevar a cabo un proceso comunicativo de calidad. Martínez (2016) y Montero \& Sanz (2008) centran, además, su acción en un ir y venir continuo de la teoría a la práctica, en la asunción de procesos reflexivos que permitan el surgimiento de dudas, pensamientos e incluso paradojas educativas, asumiendo como propio el enfoque de la investigación-acción.

Ante esta conceptualización, ¿cuáles son las tareas concretas asociadas a la función asesora?

\section{Las tareas del profesor-asesor}

Autores como Ainscow, Bereford, Harris, Hopkins \& West (2001) y Lago \& Onrubia (2011) consideran dos perspectivas que ayudan a definir las diversas tareas del profesor-asesor:

1) La perspectiva sobre cómo interviene con los estudiantes. Tareas que se desprenden de procesos de autoanálisis y reflexión, de construcción conjunta, de establecimiento de acuerdos, etc.

De acuerdo con Imbernón (2007), esto incide en el papel de amigo crítico (según parámetros epistemológicos de Eisner, 1985), mediando con base en procesos de reflexión crítica en la búsqueda de soluciones por parte de los estudiantes. Esto supone considerar como ámbitos de dominio propios del profesor-asesor la competencia comunicativa, el conocimiento de la práctica, la negociación, el conocimiento de técnicas orientadas al diagnóstico, al análisis de necesidades y la toma de decisiones. 
Esta perspectiva, unida al área de apoyo emocional (Hennissen et al., 2011), permite considerar la figura de un profesor-asesor preocupado por aportar la seguridad y confianza necesarias para que los estudiantes puedan generar conocimiento en y desde las nuevas situaciones a las que se enfrentan.

2) La perspectiva relacionada con en qué intervienen: qué conocimientos se abordan. Esta perspectiva se vincula con el área de asistencia a la tarea (Hennissen et al., 2011), que permite situar al docente en un proceso de ayuda a la adquisición y perfeccionamiento de competencias profesionales, utilizando estrategias como la retroalimentación, la información y los consejos sobre la práctica.

Las aportaciones de Montero \& Sanz (2008) y Hargreaves \& Fink $(2002,2006)$ plantean la necesidad de mantener una visión sistémica y compleja, considerando el clima y la cultura profesional y teniendo presente tanto la diversidad como la riqueza de los distintos entornos.

\section{El saber experto del profesor-asesor}

Parece ampliamente superada la idea del profesor-asesor como aquel experto que dirige su acción a proporcionar soluciones que desde la generalidad pretendan dar respuesta a los problemas educativos y profesionales (Pérez, 2016). Sin embargo, no debe olvidarse que él es poseedor de un saber experto nacido de la propia práctica profesional, de su experiencia en continuo ir y venir con la teoría, "como conocimiento profundo y analítico de lo empírico en un interjuego dinámico con la teoría" (Nicastro \& Andreozzi, 2006, p. 24). Este conocimiento debe ser utilizado para el diagnóstico de situaciones, necesidades y motivaciones, y debe integrar las condiciones profesionales, sociales y culturales con base en las cuales diseñar acciones formativas (Herrera-González, 2010; Monereo \& Pozo, 2005; Pérez, 2016; Schleicher, 2016).

Existe, no obstante, cierta tensión entre las diferentes consideraciones defendidas por diversos autores (Gairín, 2007; Nicastro \& Andreozzi, 2006; Pérez, 2016; Rodríguez et al., 2004). Ante la disyuntiva de mostrarse o no como un "experto infalible", surge como elemento integrador la idea de Imbernón (2007) acerca de que el profesor-asesor debe tener la capacidad de, y puede, ejercer dicho papel cuando sea necesario para salvar un obstáculo diagnosticado, nunca para dar una solución uniforme. Se debe huir, pues, del modelo de adquisición de servicios para acercarnos al modelo de asesoramiento de proceso o colaborativo, en el que el docente actúa desde la colaboración para diagnosticar obstáculos y ayudar a los demás a encontrar su propia y contextualizada solución (Pérez, 2016). 


\section{Método}

Frente a la predominante asunción de un papel transmisor por parte del profesorado universitario, poco integrador de la voz del estudiantado y con escasos recursos para integrar las herramientas necesarias que permitan una eficaz gestión personal de la carrera, surge la necesidad y finalidad de esta investigación de indagar sobre la figura del profesor-asesor como respuesta a las exigencias de una formación universitaria orientada a facilitar el desarrollo profesional de los graduados.

Se ha optado por un enfoque cualitativo de carácter comprensivo, otorgando protagonismo a las voces de los principales agentes del fenómeno abordado (Creswell, 2009; Flick, 2012), puesto que permite vislumbrar "cómo las personas experimentan, interpretan y reconstruyen los significados intersubjetivos" (Del Rincón, Latorre, Arnal \& Sans, 1995).

Para Martínez (2007, p. 31), la metodología interpretativa se basa en "teorías y prácticas de interpretación que buscan comprender lo que ocurre en diferentes contextos humanos en función de lo que las personas interpretan sobre ellos y los significados que otorgan a lo que les sucede".

En esta investigación, el enfoque cualitativo de carácter comprensivo permitirá generar y construir nuevas interpretaciones y significados con base en las experiencias de vida de alumnado y profesorado, de manera que se definan las necesidades formativas docentes y el papel de profesor-asesor.

Para alcanzar dicha finalidad, los objetivos planteados han sido:

1. Conocer, desde el punto de vista de estudiantes y profesores, las necesidades formativas del docente universitario actual para la contribución al desarrollo profesional de los graduados.

2. Delimitar y precisar el perfil del profesor-asesor universitario para favorecer la gestión personal de la carrera de los graduados.

\section{Contexto del estudio}

La investigación se ha desarrollado en dos contextos distintos, con informantes clave, pertenecientes a diferentes colectivos implicados, pretendiendo de este modo obtener una aproximación más real y compleja al fenómeno de estudio: la figura del profesor-asesor en la universidad actual.

En el primer escenario tuvo lugar una experiencia de asesoramiento a profesorado de la Universidad Politécnica de Cataluña (UPC) por parte de docentes muy bien preparados que asumieron el papel de asesor. El segundo escenario lo configuraron estudiantes de grado de la Universidad de Barcelona (UB). El objetivo fue comprender e interpretar las vivencias, experiencias y significados de las personas implicadas. Estas dos universidades están ubicadas en la ciudad de Barcelona y por su idiosincrasia abarcan 
la totalidad de carreras que se ofertan en el contexto español, y por ende aportan una visión del conjunto de docentes y estudiantes que representan las aulas en España. Además, estas son instituciones de reconocido prestigio, con muchos años de historia y presenciales; es decir que son similares en organización y estructura, lo que permite explorar y extrapolar resultados de una a la otra.

\section{Participantes y criterios de selección}

Dada la finalidad de la investigación se seleccionaron 14 profesores universitarios, que voluntariamente fueron asesorados por dos docentes expertos, y 26 egresados de universidad.

Tabla 1

Información del profesorado asesorado

\begin{tabular}{|c|c|c|c|c|c|}
\hline $\begin{array}{l}\text { Docente asesorado/ } \\
\text { código de } \\
\text { investigación }\end{array}$ & $\begin{array}{l}\text { Vinculación } \\
\text { con la } \\
\text { universidad }\end{array}$ & Género & $\begin{array}{l}\text { Asesor de } \\
\text { referencia }\end{array}$ & $\begin{array}{l}\text { Codificación } \\
\text { (entrevistas) }\end{array}$ & $\begin{array}{l}\text { Codificación } \\
\text { (grupos de } \\
\text { discusión) }\end{array}$ \\
\hline P1 & Asociado & M & A1 & $\mathrm{E} 1+\mathrm{E} 2$ & GD1 \\
\hline P2 & Colaborador & $M$ & A1 & $E 1+E 2$ & GD1 \\
\hline P3 & Asociado & $\mathrm{M}$ & A1 & $E 1+E 2$ & GD1 \\
\hline P4 & Asociada & $\mathrm{F}$ & A1 & $E 1+E 2$ & GD1 \\
\hline P5 & Asociado & $M$ & A1 & $E 1+E 2$ & GD1 \\
\hline P6 & Colaborador & $\mathrm{M}$ & A1 & $E 1+E 2$ & GD1 \\
\hline P7 & Asociada & $\mathrm{F}$ & A1 & $E 1+E 2$ & GD1 \\
\hline P8 & Asociado & $\mathrm{M}$ & A1 & $E 1+E 2$ & GD1 \\
\hline P9 & Asociado & $\mathrm{M}$ & A1 & $E 1+E 2$ & GD1 \\
\hline P10 & Asociada & $\mathrm{F}$ & $A 2$ & $E 1+E 2$ & GD1 \\
\hline P11 & Asociada & $\mathrm{F}$ & $A 2$ & $\mathrm{E} 1+\mathrm{E} 2$ & GD1 \\
\hline P12 & Ayudante & $\mathrm{M}$ & $\mathrm{A} 2$ & $E 1+E 2$ & GD1 \\
\hline P13 & Asociado & $M$ & $\mathrm{~A} 2$ & $\mathrm{E} 1+\mathrm{E} 2$ & GD1 \\
\hline P14 & Ayudante & $\mathrm{M}$ & $A 2$ & $\mathrm{E} 1+\mathrm{E} 2$ & GD1 \\
\hline
\end{tabular}


Como puede observarse en la tabla 1, la mayoría de los participantes estaban contratados como asociados, lo que significa que ejercen como docentes en la universidad mediante contratos temporales y a tiempos parciales. Debido a la voluntad de estos profesores por realizar carrera académica, existe un gran interés en participar en programas de formación de mejora de la docencia. Este interés, unido a la falta de motivación y de tiempo del profesorado permanente, que además debe investigar y hacerse cargo de tareas vinculadas con la gestión universitaria, hace que el porcentaje del profesorado temporal sea mucho más elevado que el del profesorado estable o permanente.

\section{El proceso de selección de los docentes asesores}

Para la identificación de los dos asesores, se tuvo en consideración que tuvieran: a) amplia experiencia en docencia universitaria; b) interés por la innovación, indagación y mejora docente; c) motivación por su autoaprendizaje y formación permanente; d) capacidad de autocrítica y autoevaluación, y e) habilidades sociales que potenciasen el desarrollo social, personal y profesional de los participantes. La tabla 2 recoge la información sobre la muestra de asesores.

Tabla 2

Información de los asesores

\begin{tabular}{c|l|l|l|c}
\hline $\begin{array}{c}\text { Asesor/código } \\
\text { de investigación }\end{array}$ & Vinculación & Género & Grupo & $\begin{array}{l}\text { Codificación } \\
\text { (entrevista) }\end{array}$ \\
\hline A1 & Catedrático & M & Barcelona & E1 + E2 \\
\hline A2 & Titular & $M$ & Terrassa & E1 + E2 \\
\hline
\end{tabular}

Fuente: elaboración propia

\section{El proceso de selección de los egresados}

Los egresados se seleccionaron según los siguientes criterios: a) estar trabajando, y que este desempeño fuera acorde con su titulación, y b) llevar un tiempo determinado en el mercado de trabajo a partir de la clasificación de desarrollo profesional aportada por Riverin-Simard (1990). La tabla 3 recoge la información de los egresados seleccionados. 
Tabla 3

Información de los egresados

\begin{tabular}{|c|c|c|c|}
\hline $\begin{array}{l}\text { Egresado/código } \\
\text { de investigación }\end{array}$ & Vinculación/tiempo & Género & $\begin{array}{l}\text { Codificación } \\
\text { (entrevista) }\end{array}$ \\
\hline Eg1 & Más de 16 años & $\mathrm{F}$ & E1 \\
\hline Eg2 & Entre 7 y 10 años & $\mathrm{F}$ & E1 \\
\hline Eg3 & Más de 16 años & M & E1 \\
\hline Eg4 & Más de 16 años & M & E1 \\
\hline Eg5 & Entre 11 y 15 años & $\mathrm{F}$ & E1 \\
\hline Eg6 & Entre 7 y 10 años & $\mathrm{F}$ & E1 \\
\hline Eg7 & Entre 7 y 10 años & $\mathrm{F}$ & E1 \\
\hline Eg8 & Entre 11 y 15 años & M & E1 \\
\hline Eg9 & Más de 16 años & $\mathrm{F}$ & E1 \\
\hline Eg10 & Entre 11 y 15 años & M & E1 \\
\hline Eg11 & Más de 16 años & $\mathrm{F}$ & E1 \\
\hline Eg12 & Más de 16 años & M & E1 \\
\hline Eg13 & Más de 16 años & M & E1 \\
\hline Eg14 & Entre 7 y 10 años & $\mathrm{F}$ & E1 \\
\hline Eg15 & Entre 7 y 10 años & $\mathrm{F}$ & E1 \\
\hline Eg16 & Entre 11 y 15 años & $\mathrm{F}$ & E1 \\
\hline Eg17 & Entre 11 y 15 años & M & E1 \\
\hline Eg18 & Más de 16 años & M & E1 \\
\hline Eg19 & Más de 16 años & $\mathrm{F}$ & E1 \\
\hline Eg20 & Entre 11 y 15 años & $\mathrm{F}$ & E1 \\
\hline Eg21 & Entre 7 y 10 años & $\mathrm{F}$ & E1 \\
\hline $\operatorname{Eg} 22$ & Más de 16 años & $\mathrm{F}$ & E1 \\
\hline Eg23 & Entre 7 y 10 años & $\mathrm{F}$ & E1 \\
\hline Eg24 & Más de 16 años & $\mathrm{F}$ & E1 \\
\hline Eg25 & Entre 11 y 15 años & $\mathrm{F}$ & E1 \\
\hline Eg26 & Entre 7 y 10 años & $M$ & E1 \\
\hline
\end{tabular}

Fuente: elaboración propia

\section{Recolección de la información}

Fueron dos los instrumentos de recolección de información, los cuales, de forma complementaria, permitieron abordar la finalidad y los objetivos de la investigación.

El primero fue una entrevista semiestructurada, que permitió recoger información sobre acontecimientos y aspectos subjetivos de las personas, como creencias y actitudes, opiniones, valores o conocimientos, los cuales de otro modo no estarían al alcance del investigador (Flick, 2012). Para esto se utilizaron preguntas abiertas, las cuales permitieron captar la riqueza y los matices de la información, a la vez que entrelazar temas y construir un conocimiento holístico y comprensivo de la realidad. 
Las entrevistas llevadas a cabo tuvieron como objetivos conocer y profundizar en la experiencia vivida durante el proceso formativo, así como en la valoración de los participantes con respecto al asesoramiento como modelo de formación en la universidad actual.

La investigación llevó a cabo un total de 52 entrevistas: las realizadas a profesores universitarios y a los dos asesores se hicieron en momentos diferentes de la investigación: durante su desarrollo y al finalizar, de forma que se alcanzó un total de 28 entrevistas realizadas a profesores universitarios y 4 a asesores. Asimismo, se realizaron 26 entrevistas a egresados.

El segundo instrumento fue un grupo de discusión, que permitió la interacción para hacer emerger la experiencia del grupo y así construir conjuntamente nuevas interpretaciones y significaciones sobre un mismo fenómeno. Siguiendo a Flick (2012), se promovió la apertura entre los participantes y se generó un discurso grupal para identificar distintas tendencias y regularidades en sus opiniones.

La investigación integró dos grupos de discusión (durante su desarrollo y al finalizar) dirigidos a recoger las vivencias y experiencias de los docentes que participaron bajo el rol de asesorados. Los objetivos definidos fueron:

1) Profundizar en las diferencias que los participantes identifican entre el modelo de formación tradicional y el basado en el asesoramiento.

Para ello, se pretendía descubrir qué diferencias identificaba el profesorado entre el modelo de formación basado en el asesoramiento y el modelo tradicional, qué ventajas e inconvenientes habían encontrado en el asesoramiento, la posibilidad de incluir dicho modelo en la formación inicial docente del profesorado, así como sus potencialidades a corto y largo plazo. También se pretendía interpretar cómo concebían la relación con su asesor a lo largo de todo el proceso, la resolución de dudas, la aportación de elementos de interés y para la reflexión, los factores que les ayudaron y qué características debía tener un buen asesor según su propia experiencia.

2) Analizar la concepción del profesor-asesor y la función atribuida a él.

Sobre todo al finalizar la formación mediante asesoramiento, se pretendió que el profesorado participante aportase la idea que tenían con respecto a la figura del profesor-asesor, en contraposición con la idea que tenían antes de iniciar el proceso. Definir el papel fue en todo momento de la mano con la atribución de las funciones, entendidas como aquellos objetivos vehiculados a través de acciones que se debían desarrollar al inicio, durante y al finalizar la acción formativa.

En la aplicación de los dos tipos de instrumentos se distinguieron dos fases: la prueba piloto y el diseño final. Ambos instrumentos fueron 
sometidos a un juicio de expertos (cuatro profesionales del campo de estudio, tanto a nivel de contenido como metodológico) y posteriormente se realizaron dos entrevistas a modo de prueba. A partir de las mejoras sugeridas, se elaboraron los instrumentos definitivos. Las entrevistas se grabaron con el consentimiento de los participantes. La duración media de estas fue de entre 40 y 70 minutos. En el caso de los grupos de discusión, la duración media estuvo alrededor de los 90 minutos. El procedimiento tomado para el desarrollo de los instrumentos contó con dos momentos esenciales: a) valoración de las decisiones tomadas para la panificación de la entrevista y el grupo de discusión y b) valoración del desarrollo de la entrevista y el grupo de discusión. Toda la información recogida fue transcrita literalmente, siguiendo los códigos con respecto a los informantes, instrumentos y documentos ya explicados en las tablas previas.

\section{Análisis de la información}

El proceso de categorización y análisis de datos fue cíclico, dinámico, flexible y comprensivo, de modo que se pudiera realizar una aproximación a la realidad objeto de estudio de forma deductiva e inductiva. Para ello, fue importante categorizar, codificar y clasificar los datos en función de los criterios que se establecieron para analizar e interpretar adecuadamente los significados de los comentarios emitidos por los sujetos (Martínez, 2007; Pérez, 2016). Para asegurar la calidad del proceso, se atendió esencialmente a la reflexividad. Además, se realizó una triangulación entre investigadores, en el análisis de datos, buscando la coherencia entre los resultados y las narraciones de los participantes.

Este trabajo forma parte de un estudio más amplio y complejo. La tabla 4 recoge las categorías de este artículo y su relación con los objetivos y los informantes clave.

La categoría de necesidades formativas se centró en conocer aquellas carencias que el profesorado universitario manifestaba según los egresados y según los propios docentes. Se trataba de necesidades formativas de la figura de profesor-asesor en relación con la caracterización de la propia investigación. Esta categoría fue abordada fundamentalmente en las entrevistas semiestructuradas y, de manera espontánea, en los grupos de discusión.

La categoría de relación didáctica fue una dimensión emergente que surgió fundamentalmente a partir de las entrevistas tanto a egresados como al profesorado universitario. Esta categoría especificaba la forma, el momento, el contenido y el proceso que debía seguir una relación entre profesorado y alumnado para abordar con eficacia aprendizajes vinculados con la GPC. 
Tabla 4

Especificidad de la investigación

\begin{tabular}{|c|c|c|c|}
\hline Objetivos del estudio & Categorías & Definición & $\begin{array}{l}\text { Informantes } \\
\text { clave }\end{array}$ \\
\hline \multirow[t]{2}{*}{$\begin{array}{l}\text { Conocer, desde el punto } \\
\text { de vista de estudiantes y } \\
\text { profesores, las necesidades } \\
\text { formativas del docente } \\
\text { universitario actual para la } \\
\text { contribución al desarrollo } \\
\text { profesional de los graduados }\end{array}$} & $\begin{array}{l}\text { Necesidades } \\
\text { formativas }\end{array}$ & $\begin{array}{l}\text { Informa sobre las } \\
\text { características actuales } \\
\text { del docente universitario, } \\
\text { considerando tanto sus } \\
\text { carencias docentes como las } \\
\text { necesidades formativas }\end{array}$ & $\begin{array}{l}\text { Profesorado } \\
\text { universitario } \\
\text { asesorado y } \\
\text { egresados }\end{array}$ \\
\hline & Relación didáctica & $\begin{array}{l}\text { Dimensión emergente del } \\
\text { estudio. Informa sobre cómo } \\
\text { la relación didáctica es clave } \\
\text { para la GPC }\end{array}$ & $\begin{array}{l}\text { Profesorado } \\
\text { universitario } \\
\text { asesorado y } \\
\text { egresados }\end{array}$ \\
\hline \multirow[t]{2}{*}{$\begin{array}{l}\text { Delimitar y precisar el perfil del } \\
\text { profesor-asesor universitario } \\
\text { para favorecer la gestión } \\
\text { personal de la carrera de } \\
\text { los graduados }\end{array}$} & $\begin{array}{l}\text { Características del } \\
\text { profesor-asesor }\end{array}$ & $\begin{array}{l}\text { Informa sobre las } \\
\text { características que debe tener } \\
\text { el docente universitario para } \\
\text { poder ejercer positivamente la } \\
\text { función de profesor-asesor }\end{array}$ & $\begin{array}{l}\text { Profesorado } \\
\text { universitario } \\
\text { asesorado y } \\
\text { asesores }\end{array}$ \\
\hline & $\begin{array}{l}\text { Funciones del } \\
\text { profesor-asesor }\end{array}$ & $\begin{array}{l}\text { Informa y detalla las acciones } \\
\text { que debe llevar a cabo el } \\
\text { profesor para desarrollar su } \\
\text { papel de profesor-asesor en } \\
\text { la universidad }\end{array}$ & $\begin{array}{l}\text { Profesorado } \\
\text { universitario } \\
\text { asesorado, } \\
\text { asesores y } \\
\text { egresados }\end{array}$ \\
\hline
\end{tabular}

Fuente: elaboración propia

La categoría de características del profesor-asesor apareció en las entrevistas a egresados, pero fue abordada en profundidad en los grupos de discusión. Esta dimensión pretendía recoger las percepciones de alumnado y profesorado acerca de los rasgos que debían definir a un buen profesorasesor. Dichos rasgos se centraban fundamentalmente en atributos personales y académicos.

La categoría de funciones del profesor-asesor fue importante, ya que, en parte, recogía y daba coherencia a las categorías anteriormente mencionadas. Esta integra todas aquellas acciones atribuidas al profesor-asesor en relación con el conocimiento académico, el conocimiento didáctico y la forma de establecer contacto con el alumno con el fin de facilitarle sus aprendizajes. Dicha categoría bebió fundamentalmente de los grupos de discusión, aunque fue matizada con las aportaciones de los egresados durante las entrevistas, puesto que las necesidades que manifestaban apuntaban a funciones claramente necesarias para su orientación académica y profesional. 


\section{Resultados}

Atendiendo a los objetivos y categorías de la investigación, se presentan a continuación los principales resultados. Antes de adentrarnos en ellos, hay que señalar que su descripción surge a partir del análisis de la información entregada por los tres agentes clave de la investigación y que los autores del estudio centramos la mirada en aquellos elementos recurrentes a lo largo de las entrevistas y en el grupo de discusión.

\section{Las necesidades formativas del profesorado universitario actual para contribuir con el desarrollo profesional de sus estudiantes}

Los docentes coinciden en señalar la existencia de cuestiones concretas vinculadas a las necesidades formativas (categoría de análisis) del futuro docente universitario, las cuales dependerán del momento de su trayectoria profesional; por tanto, el factor tiempo dedicado a la enseñanza es determinante para poder poner en situación ciertas competencias profesionales.

El primer elemento es poder disponer de formación didáctica-pedagógica. Los docentes consideran que la profesión de docente universitario no está regulada y que el acceso puede ser desigual dependiendo de la disciplina en la que se sitúen, por ello sería necesario asegurar formación o capacitación en: a) mejorar el aprendizaje mediante metodologías activas, b) saber comunicar bien, c) obtener herramientas de evaluación adecuadas a la diversidad de estudiantes, d) planificar y gestionar procesos formativos, e) conocer otras experiencias docentes para enriquecer el aprendizaje continuado, f) tener mayor conocimiento de la institución y las implicaciones que ello conlleva en el ejercicio de la profesión y g) actualizarse permanentemente. Así como hay una formación en investigación a partir del doctorado, sería importante poder asegurar una formación en tareas docentes. A continuación, se presenta una cita que resume la idea planteada por la mayoría de los egresados y el profesorado entrevistado.

Hacer que mis clases sean más amenas y que todos los alumnos participen más y no sean siempre los mismos quienes intervienen en clase (P9E1). De alguna manera al inicio del ejercicio profesional es vital conectarse con los estudiantes, generar un buen clima de aula y potenciar procesos de enseñanza-aprendizaje significativos (Eg1).

En cambio, cuando ya disponen de una sólida formación docente (no al inicio del ejercicio profesional), los entrevistados señalan que los docentes deben empezar a considerar otras necesidades formativas: a) creación de materiales, b) fomento y adquisición de competencias transversales por 
parte de los alumnos, c) utilización de recursos tecnológicos para la comunicación didáctica, d) fomento de la creatividad del alumnado y su motivación, e) investigación educativa, f) contribuir con la identidad profesional de los estudiantes y g) la creación de su propia carpeta docente.

Además de las necesidades expuestas, para más de dos tercios de los egresados, la relación didáctica (categoría surgida en la investigación) establecida con el docente ha sido determinante durante en su proceso de aprendizaje. Esta es una categoría con identidad propia, y por ello se presenta por sí sola. Se concibe como un trabajo que se cuide desde el inicio de la docencia, ya que es la que propicia que todo lo anterior fluya. Los entrevistados la han definido como la capacidad que tiene el docente para despertar el interés, invitar al cuestionamiento, cuidar la comunicación con el estudiante y facilitar espacios y recursos para que se construyan y crezcan como profesionales. En su recuerdo, aludían a casos puntuales de buenos profesores, que cumplían con las características descritas. Este elemento del proceso formativo es trascendental para los estudiantes, por lo que se le otorga entidad propia, dada su relevancia en la construcción de su carrera.

Alguna profesora, hay unos referentes profesionales como XXX, que yo la quiero un montón, era una persona que yo, casi de entrada me despertaba, porque era una provocadora nata en las clases y a mí me hizo, realmente me provocó y me hizo actuar (P5E2, Eg15).

De hecho, más de la mitad de los egresados (en especial los que llevaban entre siete y diez años en el mercado de trabajo) fueron críticos con esta cuestión analizada: "La carrera y sobre todo algunos profesores en concreto deben inculcarte ser profesional, salir de la carrera acostumbrado a preguntarte y cuestionarte cosas" (P7E1). "Conectarte con la profesión y apostar por ella" (Eg18). En concreto, los egresados expresaron que había pocos profesores que fueran capaces de crear un vínculo efectivo entre relación y formación profesional para ayudarlos a encontrar su camino profesional, y consideraron vital que se incluyera esta dimensión en la universidad. De hecho, es de resaltar que esta cuestión tan necesaria para los egresados no haya sido un elemento para considerar por parte de los docentes universitarios cuando piensan en sus necesidades formativas, aunque sí aluden a la relación con los estudiantes, pero considerándola únicamente como una cuestión para mejorar metodologías de enseñanza, la comunicación en el aula y los procesos evaluativos. 


\section{La figura del profesor-asesor en la universidad del siglo XXI}

La primera categoría analizada al respecto fue cuáles son las características que debe tener el profesor-asesor universitario en la actualidad. Después de la experiencia vivida por un grupo de docentes universitarios (asesores y asesorados), sus reflexiones alrededor de esta figura en la formación universitaria determinó, en primer lugar, que este debe comprender a los estudiantes y adelantarse a lo que estos pueden encontrar al ejercer su profesión, para ello debe haber vivido situaciones profesionales que le doten de conocimientos teóricos y prácticos relevantes.

Habría que pedirle que tuviera una cierta trayectoria en haberse enfrentado con el proceso de probar cosas y reflexionar sobre ellas, yo creo que eso es inevitable (...), y tener un cierto conocimiento de recursos al alcance de la gente para documentarse (P11E2, A1E1, P7GD1).

Pero formación y experiencias profesionales, aun siendo importantes, no son suficientes. El elemento imprescindible para que la acción asesora pueda llevarse a cabo con éxito es la motivación por ejercerla: "Básica y principal: querer hacerlo" (A2E2).

De hecho, esta motivación podrá hacer que cualquier profesor-asesor, a lo largo de los años, continúe con ganas de mejorar la enseñanza en sus clases y de proponer nuevas metodologías para el aprendizaje a sus estudiantes. Es fundamental mantenerse activo para la búsqueda de estrategias, recursos y actualización pedagógica del ámbito de estudio en el que el docente es experto. Continuamente ha de estar mejorando su función como docente y conectando con los nuevos estudiantes que, año a año, ingresan a su aula.

Existen otros elementos distintos a los señalados anteriormente, también relevantes, como: a) reconocer las inquietudes docentes y profesionales; que sepa detectar y potenciar las capacidades y competencias de los estudiantes; b) tener conocimiento sobre aquello que es fundamental para la formación y para el mercado laboral; que organice y paute a la vez que es crítico con el trabajo de los estudiantes; c) tener orientación a ayudar y dar apoyo durante el proceso formativo y preprofesional, y d) tener visión holística del programa formativo y de la profesión, a la vez que se orienta a las necesidades y particularidades individuales y grupales: "Es alguien que te acompaña durante toda la formación y que te ayuda a darle una visión de conjunto a las cosas" (P1GD1, P12, A1E1).

Desde el punto de vista concreto de los docentes asesorados, las características fundamentales del asesor deben ser: tener experiencia docente y profesional, estar motivado para ejercer la función asesora, tener 
la capacidad de comunicar y escuchar, así como una actitud orientada al aprendizaje de los estudiantes.

En cuanto a la otra categoría, funciones propias del profesor-asesor universitario, desde una concepción dialógica e integrando la visión de todas las personas implicadas, se encuentran la siguientes:

Tener motivación para crear una relación pedagógica adecuada con los estudiantes, para lo cual es fundamental conocerlos. Partir de su realidad, su contexto próximo, su situación vital previa para propiciar la adaptación metodológica necesaria para conectar con los estudiantes e iniciar la acción asesora. Además, atender las vicisitudes de cada uno para adecuarlas al grupo será vital.

Tener un asesor (...) que desde el inicio te vea, sepa tus dudas, vea tu asistencia y vea cómo vas evolucionando. Tener una persona a la que puedas recurrir para consultas y que sepa de tu evolución (P5GD1).

Se necesitan profesores que te ayuden a construir, que te cuiden, que se impliquen, que estén conectados con el mundo laboral y te conecten (Eg6).

El aprendizaje se promueve más fácilmente si el profesor-asesor consigue atender unas premisas previas al inicio de la docencia: a) crear un clima adecuado en el aula para que los estudiantes puedan sentirse cómodos en el continuo cuestionamiento de lo enseñado; b) atender a los elementos clave de la formación (con relación a sus clases) y reforzarlos como vitales para que el estudiante pueda comprender que son fundamentales para su futuro profesional; c) incentivar en sus estudiantes el gusto por el análisis y la reflexión de todo lo que van aprendiendo en su nueva etapa, dos características fundamentales a adquirir a lo largo de su ejercicio como profesionales, y d) continuamente proporcionar retroalimentación de las tareas — desde el sentido de la evaluación continuada - para que los estudiantes puedan analizar sus avances, límites y oportunidades de mejora:

Él lo tendría que tener muy claro (...) cuáles son los conocimientos básicos que debemos adquirir y cómo distribuirlos a lo largo del curso, porque por muchas inquietudes que tenga el alumno, él debe saber conducirnos al aprendizaje esperado (A2E2).

El docente debe motivarnos en la asignatura y ser entusiasta para engancharnos con la docencia, con el contenido de la materia, debe ser el que promueva este proceso $(\mathrm{Eg} 7)$. 
Además, el profesor-asesor debe favorecer, guiar y acompañar las experiencias académicas y profesionales, ayudando a que estas se conviertan en fuente de aprendizaje (sobre todo durante la puesta en práctica de los aprendizajes). Para ello debe: a) motivar, animar y promover la acción de los asesorados; b) hablar abiertamente de los obstáculos que pueden encontrarse y proporcionar recursos para seguir adelante, y c) preparar para la futura carrera profesional.

Ha sido capaz de plantear aspectos que yo ni había pensado o situaciones que supongo que ha vivido en el aula que yo no había pensado y me ha dado una guía de cómo afrontar ciertas situaciones (P4E2).

Necesitamos docentes que generen estudiantes activos, que antes de acabar ya se espabilen y tengan salidas profesionales, que sepan qué quieren hacer si trabajar o seguir formándose con una actitud más proactiva, más de emprendeduría, más de generar crecimiento profesional (Eg20).

Así, el profesor-asesor debe promover el aprendizaje colaborativo y el intercambio de experiencias entre iguales. Para ello es un fundamental el aprendizaje reflexivo. En este sentido es necesario: a) desarrollar y dinamizar las actividades con el fin de favorecer la relación entre iguales y b) abrir espacios para que los estudiantes expongan y expliquen sus experiencias al resto de compañeros, ofreciendo la posibilidad de plantearse preguntas entre ellos.

También debe poner a disposición de los estudiantes su propia experiencia profesional y basarse en el conocimiento adquirido de esa experiencia, la cual le hace comprender la situación en la que se encuentran los estudiantes, aportándole una fuente y un cuerpo de conocimiento en sí misma.

Creo que el más importante es notar que él tiene mucha experiencia, es decir, el hecho de que su experiencia esté disponible y que cualquier problema que tienes tú sabes que lo responde con su experiencia, es muy importante, y se nota en las respuestas que da en general y las que te da a ti concretamente con tu problema. Esto es [lo] que más me ha ayudado (P7E2).

Atendiendo a lo comentado por el A2, una de las funciones del profesor-asesor en los procesos formativos es conseguir que los estudiantes naveguen en aguas turbulentas, es decir, tensionados por el propio proceso. No es adecuado no sentir frustración, miedos o desasosiego en ningún momento, porque la calma le puede llevar a la desmotivación. Ahora bien, 
esa tensión tiene que ser graduada, para que sean capaces de observar que aprenden y progresan en su vida académica, de lo contrario la frustración puede paralizar el proceso formativo. Por tanto, hay que saber cuándo aportar inquietud y cuando tutorizar para acompañarlos en su trayectoria académica: "La intención ha de ser la de ir involucrando, la de mantener a las personas involucradas y reflexivas y activas sobre todo lo que se está haciendo" (A2E1).

Favorecer la profesionalización, contribuyendo a buscar el arraigo entre profesión y mercado de trabajo y universidad: "Trabajar desde el inicio el estilo personal de cada uno y sus cualidades, es decir, conectar su yo personal con su yo profesional (...) ir gestando la identidad profesional del estudiante para encontrar sentido a su profesión" (P7GD1).

\section{Conclusiones}

Tras el análisis de los resultados, y atendido a los objetivos del estudio, las conclusiones se presentan a partir de dos ejes que han transcendido de la investigación: la formación docente a partir de la necesidad de los agentes intervinientes y lo que se ha de considerar en la preparación de esta figura profesional como profesor-asesor en la actual universidad.

Existe en gran parte de la comunidad académica una importante reivindicación con relación al reconocimiento que debería asumir la docencia en las instituciones universitarias y en las agencias oficiales responsables de la acreditación docente, hoy día muy centradas en otorgar un mayor grado de importancia a la tarea investigadora (Badia-Pujol, 2012). El reconocimiento de la docencia pasa por otorgar valor, sentido, coherencia y cientificidad a la formación docente del profesorado universitario. Es por ello que las instituciones y sistemas universitarios deben asumir de pleno su responsabilidad y obligación en la formación del profesorado (Imbernón, 2007; Jarauta-Borrasca \& Medina-Moya, 2009; Pérez, 2016). Esto conlleva no solo diseñar, organizar y gestionar acciones formativas, como ya hacen unidades como los institutos de ciencias de la educación o similares, principalmente para el profesorado novel, sino también reconocerlas y otorgarles el valor que merecen para la función docente y la calidad de la enseñanza universitaria (Estévez-Nenninger et al., 2014; Pérez, 2016).

Muchos estudios amparan la idea de una preparación mínima previa a la acción docente, que debería ser objeto de un debate y análisis en profundidad en el seno de la universidad española (Martínez, 2016), en la que deberían participar tanto el profesorado como los responsables y otros agentes implicados en la formación y mejora de la carrera académica (Pérez, 2016). 
Ante la premisa de la necesidad de una formación básica obligatoria para el ejercicio de la función docente universitaria, una de las preguntas que cabe plantearse es ¿qué elementos debe considerar dicha formación?

Partiendo de las necesidades que los docentes universitarios en activo dicen tener, así como de las carencias que los estudiantes egresados identifican en el profesorado que le formó para su actual profesión, este estudio identifica un elemento en común: el profesorado de hoy requiere de mayor formación para el establecimiento de la relación didáctica; relación que le debería permitir ayudar a los estudiantes en su proceso de aprendizaje y en su preparación para el ejercicio de la profesión (Fraser, 2016; Nicastro \& Andreozzi, 2006).

Pero de la comparación entre necesidades sentidas y carencias identificadas emerge un aspecto de gran importancia para la GPC desde el punto de vista de los egresados: el docente universitario requiere de un mayor contacto con la profesión, aspecto que en ningún caso aparece entre los docentes, quienes centran sus necesidades en tareas más próximas al campo didáctico y pedagógico. ¿Debería pues la formación docente integrar experiencias profesionales de las cuales los profesores puedan aprender con la finalidad de trasladarlas a la formación de sus estudiantes?

El vínculo entre teoría y práctica, la actualización de contenidos desde el ámbito profesional, la asimilación de conocimientos mediante ejemplos, situaciones, escenarios y problemáticas propias de la profesión son reivindicados por los estudiantes en su doble preocupación por disponer de los conocimientos y competencias necesarias, a la vez que estas les sirvan durante sus posteriores años de ejercicio profesional.

Hay, por tanto, cuestiones para cambiar sobre la docencia para poder articular procesos de enseñanza-aprendizaje significativos. Los profesores han de favorecer la profesionalización, contribuyendo a buscar el arraigo entre profesión, mercado de trabajo y universidad. Estévez-Nenninger et al. (2014) y Pérez \& Serrano (2012) remarcan que una de las misiones de la universidad es la preparación de los profesionales para el siglo XXI, y ello repercute en la idea del docente como articulador y no como experto infalible que transmite conocimientos.

Desde sus inicios, la formación docente del profesorado universitario se ha preocupado mucho en integrar contenido didáctico-pedagógico. Sin embargo, hoy día también surge con fuerza la necesidad de considerar el conocimiento didáctico de contenido en los términos acuñados por Shulman (2005). Además, es hora de empezar a considerar en esta formación la creación de espacios y escenarios donde la profesión para la que preparan a sus estudiantes tenga entidad propia, acercando así los postulados de la GPC a las aulas universitarias (Pinto et al., 2015). 
Han sido muchos los autores que han pretendido concretar una serie de rasgos propios de la acción asesora del docente universitario, entre los que cabe destacar los trabajos de Montero \& Sanz (2008) y Nicastro \& Andreozzi (2006). Un análisis dialógico de la literatura existente y los resultados de esta investigación conducen a establecer las siguientes características principales para el profesor-asesor: a) tener dominio de conocimientos sobre docencia (Imbernón, 2007; Monereo \& Pozo, 2005; Schleicher, 2016), b) tener estrechos vínculos con la profesión para la que prepara a sus estudiantes, orientándose a facilitar la GPC (Lent \& Brown, 2013), c) tener actitud de implicación y compromiso, predisposición y disponibilidad (Nicastro \& Andreozzi, 2006) y d) tener competencia interpersonal y comunicativa (Rodríguez et al., 2004; Sánchez-Tarazaga, 2016).

Del estudio llevado a cabo, y bajo la atenta mirada a las aportaciones que al respecto realizan autores como Montero \& Sanz (2008) y Lago \& Onrubia (2011), se desprende la necesidad de que el profesor-asesor asuma como funciones propias: a) conocer a los participantes, así como su situación y contexto; b) facilitar el aprendizaje desde su papel docente; c) favorecer, acompañar y aprender de las experiencias académicas y profesionales; d) promover el aprendizaje colaborativo y el intercambio de experiencias entre iguales; e) diseñar, desarrollar y evaluar el proceso de formación para su posible mejora; f) poner a disposición de los estudiantes su propia experiencia como profesional; g) atender de forma individualizada; h) ayudar a la interrelación del estudiante en el marco del contexto institucional, e i) mantener la tensión a lo largo de todo el proceso formativo (Pérez, 2016).

Asumir la función asesora del docente universitario para facilitar la GPC requiere de un gran apoyo para su acción (Gairín, 2007), consistente en todo un repertorio de materiales, procedimientos, estrategias, recursos, etc., pero también de una fuerte coordinación con la institución y otros docentes que ejerzan la misma función (Monereo \& Pozo, 2005; Nicastro \& Andreozzi, 2006; Pérez, 2016). En este sentido, las instituciones universitarias necesitan de equipos docentes, áreas de coordinación y comisiones para entrelazar acciones que repercutan en la calidad de la docencia.

En este punto también es necesario señalar algún límite del estudio, como la dificultad que hubo para manejar tantísima información generada por los participantes y el sesgo derivado de los profesores asesores que participaban del programa formativo (se debería entrevistar a profesionales que hayan realizado formaciones con el objetivo de analizar su práctica docente, ampliando la muestra de asesores para tener una visión más amplia del fenómeno estudiado). Otro de los límites del estudio es que, dada su contextualización, los resultados, aunque interesantes a modo de reflexión como marco para las instituciones universitarias actuales, deben 
ser entendidos primordialmente en el contexto desde el que parten. Por lo tanto, se tendrían que realizar, además, estudios de corte cuantitativo para hacer análisis más macro y generalizables para la educación superior. Se debe seguir profundizando, por tanto, en el perfil del profesor-asesor universitario, dado que este estudio recoge datos parciales de la investigación. No obstante, también se han generado propuestas para poder implementar algunos de sus resultados, como crear espacios docentes que den apoyo a la función de la mentoría y asesoramiento en la universidad, generar sinergias entre la relación dialéctica universidad-profesión y poner en práctica en el quehacer del docente universitario los resultados de la investigación, evaluando su efectividad y eficacia en la formación de los futuros profesionales. Hay que continuar trabajando en el camino de la función docente de la universidad conectada a la formación profesional de sus estudiantes: recogiendo datos, proponiendo acciones, implementándolas y evaluándolas para asegurar la calidad formativa del profesorado y de la formación de los estudiantes. La universidad en estos últimos años se ha visto sometida a una profunda transformación, también en su labor de preparación de sus estudiantes, y por ello hay que avanzar en la propuesta de actuaciones a partir de datos contrastados, para lo que este artículo puede ayudar. Por ende, como reto a futuro está analizar qué tipo de docente universitario se necesita en las universidades en la actualidad, atendiendo a las tres funciones de su profesión: docencia, investigación y gestión. Otro de los retos que se desprende del estudio es analizar el campo de las universidades virtuales (en estos momentos en expansión), para adecuar la formación del profesor-asesor a las necesidades del estudiante en ese escenario mediatizado por las TIC en su relación didáctica. Este estudio está contextualizado en universidades presenciales y con una larga trayectoria formativa, por lo que este marco de partida condiciona la lectura de los resultados obtenidos.

\section{Sobre los autores}

María José Pérez-Cabrera es doctora en Educación y Sociedad, especializada en Asesoramiento. Ha sido responsable de la formación docente en la Universidad Politécnica de Cataluña, España, y profesora en la Universidad de Barcelona, España. Actualmente es técnica superior de Educación y diseña y desarrolla proyectos de mejora educativa municipal. Es autora de artículos y comunicaciones sobre la formación docente.

Juan Llanes-Ordoñez es profesor asociado y profesor-coordinador de la Universidad de Barcelona, España, y del Centro Universitario Internacional de Barcelona, España. Doctor en Educación y Sociedad. Miembro del grupo de 
investigación Transiciones Académicas y Laborales (TRALS) y coordinador del grupo de innovación docente consolidado Intermaster. Es director del máster en Psicopedagogía de la Universidad de Barcelona, España.

\section{Bibliografía}

Ainscow, M., Bereford, J., Harris, A., Hopkins, D. \& West, M. (2001). Crear condiciones para la mejora del trabajo en el aula. Madrid: Narcea.

Arnau, L. (2013). Desempleo y orientación para la carrera: aplicación y evaluación de un programa de adaptación a los cambios profesionales en la adultez. Educación XX1, 16(1), 191-206. doi: https://doi.org/10.5944/edu cxx1.16.1.723

Badia-Pujol, J. (2012). Ayudar al desarrollo profesional de los docentes: www. practicareflexiva. pro. Magis. Revista Internacional de Investigación en Educación, 4(9), 757-764. Recuperado de https://revistas.javeriana.edu.co/in dex.php/MAGIS/article/view/3587

Cardenal, M. E. (2006). El paso a la vida adulta. Dilemas y estrategias ante el empleo flexible. Madrid: Centro de Investigaciones Sociológicas, Siglo XXI.

Comisión Europea. (2010). Europa 2020. Una estrategia para un crecimiento inteligente, sostenible e integrador. Recuperado de http://ec.europa.eu/com mission_2010-2014/president/news/documents/pdf/20100303_1_es.pdf

Cordeiro, S., Taveira, M., Neves, L., Silva, A. Rodrigues, B. \& Costa-Lobo, C. (2017). Efeitos de uma intervenção psicológica vocacional na adaptabilidade de carreira. Revista de Psicologia da Criança e do Adolescente, 8(1), 93-104. Recuperado de https://pdfs.semanticscholar.org/2303/d7d446a6499c1ac60bb164544caf8ce01887.pdf

Creswell, J. W. (2009). Research Design: Qualitative, Quantitative, and Mixed Methods Approaches. Los Ángeles: Sage.

Del Rincón, D., Latorre, A., Arnal, J. \& Sans, A. (1995). Técnicas de investigación en ciencias sociales. Madrid: Dykinson.

Eisner, E. W. (1985). The Art of Educational Evaluation. Londres: Falmerpress.

Estévez-Nenninger, E., Valdés-Cuervo, A., Arreola-Olivarría, C. \& Zavala-Escalante, M. (2014). Creencias sobre enseñanza y aprendizaje en docentes universitarios. Magis. Revista Internacional de Investigación en Educación, 6(13), 49-64. Recuperado de https://doi.org/10.11144/Javeriana.M6-13.CSEA

Flick, U. (2012). Introducción a la investigación cualitativa. Madrid: Morata.

Fraser, J. W. (2016). La formación del profesorado en Estados Unidos. Debates y críticas. Bordón, 68(2), 35-49. Recuperado de https://doi.org/10.13042/ Bordon.2016.68203

Gairín, J. (2007). El centro como escenario educativo. En J. Bonals \& M. Sánchez (Coords.), Manual de asesoramiento pedagógico (pp. 105-142). Barcelona: Graó.

Greenhaus, J. H. \& Callanan, G. A. (1994). Career Management. Fort Worth, TX: The Dryden.

Hall, K. M., Draper, R. J., Smith, L. K. \& Bullough, R. V. (2008). More than a place to teach: Exploring the perceptions of the roles responsibilities of mentor teachers. Mentoring \& Tutoring: Partnership in Learning, 16, 328-345. Recuperado de http://dx.doi.org/10.1080/13611260802231708. 
Hargreaves, A. \& Fink, D. (2002). Sostenibilidad en el tiempo. Cuadernos de Pedagogía, 319, 16-21.

Hargreaves, A. \& Fink, D. (2006). Estrategias de cambio y mejora en educación caracterizadas por su relevancia, difusión y continuidad en el tiempo. Revista de Educación, 339, 43-58. Recuperado de http://www.ince.mec.es/ revistaeducacion/re339/re339_04.pdf

Hennissen, P., Crasborn, F., Brouwer, N., Korthagen, F. \& Bergen, T. (2011). Clarifying pre-service teacher perceptions of mentor teachers' developing use of mentoring skills. Teaching and Teacher Education, 27, 1049-1058. doi: 10.1016/j.tate.2011.03.009

Herrera-González, J. D. (2010). La formación de docentes investigadores: el estatuto científico de la investigación pedagógica. Magis. Revista Internacional de Investigación en Educación, 3(5), 53-62. Recuperado de https://www. redalyc.org/articulo.oa?id $=281023476003$

Imbernón, F. (2007). La formación y el desarrollo profesional del profesorado. Hacia una nueva cultura profesional. Barcelona: Graó.

Jarauta-Borrasca, B. \& Medina-Moya, J. (2009). La formación pedagógica inicial del profesorado universitario: repercusión en las concepciones y prácticas docentes. Magis. Revista Internacional de Investigación en Educación, 1(2), 357-370. Recuperado de https://revistas.javeriana.edu.co/index.php/MAGIS/ article/view/3390

Lago, J. R. \& Onrubia, J. (2011). Asesoramiento psicopedagógico y mejora de la práctica educativa. Barcelona: Cuadernos de Educación.

Lent, R. W. \& Brown, S. D. (2013). Social cognitive model of career self-management: Toward a unifying view of adaptive career behavior across the life, span. Journal of Counseling Psychology, 60(4), 557-568. doi: 10.1037/ a0033446

Llanes, J., Figuera, P. \& Torrado, M. (2015). Gestión personal de la carrera. Factor clave para el desarrollo profesional. En Aidipe (Ed.), Investigar con y para la acción (pp. 887-899). Cádiz: Aidipe.

Llanes, J., Figuera, P. \& Torrado, M. (2017a). Competencias de acceso y desempeño del trabajo para los graduados en Pedagogía. Revista Brasileira de Orientação Profissional, 18(2), 209-220. Recuperado de https://dx.doi. org/10.26707/1984-7270/2017v18n2p209

Llanes, J., Figuera, P. \& Torrado, M. (2017b). Desarrollo de la empleabilidad y gestión personal de la carrera de graduados en Pedagogía. Revista Española de Orientación y Psicopedagogía, 28(2), 46-60. Recuperado de https://doi. org/10.5944/reop.vol.28.num.2.2017.20118

Martínez, M. (2016). La formación inicial de los maestros: una responsabilidad compartida. Bordón, 68(2), 9-16. Recuperado de https://doi.org/10.13042/ Bordon.2016.68201

Martínez, R. A. (2007). La investigación en la práctica educativa: guía metodológica de investigación para el diagnóstico y evaluación en los centros docentes. Madrid: Meccide.

Michavila, F. \& Parejo, J. L. (2008). Políticas de participación estudiantil en el Proceso de Bolonia. Revista de Educación, número extraordinario, 85-118.

Monereo, C. \& Pozo, J. I. (Coords.). (2005). La práctica del asesoramiento educativo a examen. Barcelona: Graó. 
Montero, L. \& Sanz, M. D. (2008). Entre la realidad y el deseo: una visión del asesoramiento. Profesorado. Revista de Currículum y Formación del Profesorado, 12(1), 1-16. Recuperado de https://www.ugr.es/ recfpro/rev121COL3.pdf

Nicastro, S. \& Andreozzi, M. (2006). Asesoramiento pedagógico en acción. La novela del asesor. Buenos Aires: Paidós.

Pérez, M. J. (2016). El asesoramiento de proceso como modelo de formación inicial del profesorado universitaria (tesis doctoral). Universidad de Barcelona, Barcelona, España.

Pérez, F. \& Serrano, L. (2012). Universidad, universitarios y productividad en España. Bilbao: Fundación BBVA.

Piccolo, R. F. \& Colquitt, J. A. (2006). Transformational leadership and job behaviors: The mediating role of job characteristics. Academy of Management Journal, 49, 327-340. Recuperado de https://doi.org/10.5465/ amj.2006.20786079

Pinto, J., Taveira, M. \& Llanes, J. (2015). Cómo orientar la gestión de la carrera profesional. Barcelona: UOC.

Riverin-Simard, D. (1990). Adult vocational trajectory. The Career Development Quarterly, 39(2), 129-142. Recuperado de http://dx.doi.org/10.1002 /j.2161-0045.1990.tb00834.x

Rodríguez, J. M. et al. (2004). El asesoramiento pedagógico para la formación docente del profesorado universitario: ¿cómo profundizar en las estrategias de asesoramiento a través del cuestionario? En Pedagogía universitaria, hacia un espacio de aprendizaje compartido. III Simposio Iberoamericano de Docencia Universitaria, vol. 2 (pp. 1391-1402). Bilbao: Universidad de Deusto.

Romero, S. \& Figuera, P. (2016). La orientación en la universidad. En A. Manzanares (Ed.), Orientación a lo largo de la vida (pp. 163-196). Madrid: Wolters Kluver.

Sánchez-Tarazaga, L. (2016). Los marcos de competencias docentes: contribución a su estudio desde la política educativa europea. Journal of Supranational Policies of Education, 5, 44-67. doi: 10.15366/jospoe

Schleicher, A. (2016). Teaching excellence through professional learning and policy reform: Lessons from around the world, international summit on the teaching profession. París: OECD.

Shulman, L. (2005). Conocimiento y enseñanza: fundamentos para una nueva reforma. Profesorado. Revista de Currículum y Formación del Profesorado, 9(2), 1-30. Recuperado de https://www.ugr.es/ recfpro/rev92ART1.pdf

Sotomayor-Echenique, C., Coloma-Tirapegui, C., Parodi-Sweis, G., Ibáñez-Orellana, R., Cavada-Hrepich, P. \& Gysling-Caselli, J. (2013). Percepción de los estudiantes de pedagogía sobre su formación inicial. Magis. Revista Internacional de Investigación en Educación, 5(11), 375-392. 